\title{
Isotopic evaluation of cat diet: high variability within pet foods prevents the identification of native species in the diets of pet cats
}

\author{
Brandon W McDonald ${ }^{\text {Corresp., } 1,2}$, Troi Y Perkins ${ }^{1,2}{ }^{\text {, Robert R Dunn }}{ }^{3,4}$, Jennifer L McDonald ${ }^{5}$, Holly F Cole ${ }^{5}$, Robert S \\ Feranec $^{6}$, Roland W Kays ${ }^{1,2}$ \\ ${ }^{1}$ Department of Forestry and Environmental Resources, North Carolina State University, Raleigh, North Carolina, United States \\ 2 North Carolina Museum of Natural Sciences, Raleigh, North Carolina, United States \\ 3 Department of Applied Ecology, North Carolina State University, Raleigh, NC, United States \\ 4 Natural History Museum of Denmark, University of Copenhagen, Copenhagen, Denmark \\ 5 Center for Ecology and Conservation, University of Exeter, Penryn, United Kingdom \\ 6 New York State Museum, Albany, New York, United States \\ Corresponding Author: Brandon W McDonald \\ Email address: bwmcdona@ncsu.edu
}

Domestic cats preying on wildlife is a frequent conservation concern but typical approaches for assessing impacts rely on owner reports of prey returned home, which can be biased by inaccurate reporting or by cats consuming prey instead of bringing it home. Isotopes offer an alternative way to quantify broad differences in animal diets. By obtaining samples of pet food from cat owners we predicted that we would have high power to identify cats feeding on wild birds or mammals, given that pet food is thought to have higher $\mathrm{C}$ isotope values, due to the pervasive use of corn and/or corn by-products as food ingredients, than native prey. We worked with citizen scientists to quantify the isotopes of 202 cat hair samples and 239 pet food samples from the US and UK. We also characterized the isotopes of 11 likely native prey species from the southeastern US and used mixing models to assess the diet of 47 cats from the same region. Variation in $\mathrm{C}$ and $\mathrm{N}$ isotope values for cat food was very high, even within the same brand/flavor, suggesting that pet food manufacturers use a wide range of ingredients, and that these may change over time. Cat food and cat hair from the UK had lower $C$ values than the US, presumably reflecting differences in the amount of corn used in the food chains of the two countries. This high variation in pet food reduced our ability to classify cats as hunters of native prey, such that only $43 \%$ of the animals could be confidently assigned. If feral or free ranging cats were considered, this uncertainty would be even higher as pet food types would be unknown. Our results question the general assumption that anthropogenic foods always have high $\mathrm{C}$ isotope values, because of the high variability we documented within one product type (cat food) and between countries (US vs. UK), and emphasize the need to test 
a variety of standards before making conclusions from isotope ecology studies. 


\section{High variability within pet foods prevents the identification of native species in pet cats'}

2 diets using isotopic evaluation.

3

4
Brandon W. McDonald ${ }^{1,2}$, Troi Y. Perkins ${ }^{1,2}$, Robert R. Dunn ${ }^{3,6}$, Jennifer L. McDonald ${ }^{4}$, Holly F.

Cole $^{4}$, Robert S. Feranec ${ }^{5}$, and Roland W. Kays ${ }^{1,2}$

1. Department of Forestry and Environmental Resources, North Carolina State University, Raleigh, NC, USA

2. North Carolina Museum of Natural Sciences, Raleigh, NC, USA

3. Department of Applied Ecology, North Carolina State University, Raleigh, NC, USA

4. Center for Ecology and Conservation, University of Exeter, Penryn, U. K.

5. New York State Museum, Albany, NY, USA

6. Natural History Museum of Denmark, University of Copenhagen, 2100 Copenhagen Ø, 26 Denmark

Corresponding Author:

Brandon W. McDonald ${ }^{1,2}$

Email address: bwmcdona@,ncsu.edu 


\section{ABSTRACT}

34 Domestic cats preying on wildlife is a frequent conservation concern but typical approaches for

35

assessing impacts rely on owner reports of prey returned home, which can be biased by inaccurate reporting or by cats consuming prey instead of bringing it home. Isotopes offer an alternative way to quantify broad differences in animal diets. By obtaining samples of pet food from cat owners we predicted that we would have high power to identify cats feeding on wild birds or mammals, given that pet food is thought to have higher $\mathrm{C}$ isotope values, due to the pervasive use of corn and/or corn by-products as food ingredients, than native prey. We worked with citizen scientists to quantify the isotopes of 202 cat hair samples and 239 pet food samples from the US and UK. We also characterized the isotopes of 11 likely native prey species from the southeastern US and used mixing models to assess the diet of 47 cats from the same region. Variation in $\mathrm{C}$ and $\mathrm{N}$ isotope values for cat food was very high, even within the same brand/flavor, suggesting that pet food manufacturers use a wide range of ingredients, and that these may change over time. Cat food and cat hair from the UK had lower $\mathrm{C}$ values than the US, presumably reflecting differences in the amount of corn used in the food chains of the two countries. This high variation in pet food reduced our ability to classify cats as hunters of native prey, such that only $43 \%$ of the animals could be confidently assigned. If feral or free ranging cats were considered, this uncertainty would be even higher as pet food types would be unknown. Our results question the general assumption that anthropogenic foods always have high $\mathrm{C}$ isotope values, because of the high variability we documented within one product type (cat food) and between countries (US vs. UK), and emphasize the need to test a variety of standards before making conclusions from isotope ecology studies. 
INTRODUCTION. While the domestic cat (Felis catus) has enjoyed a status as one of

the most popular companion animals around the world, they have made a detrimental impact on wildlife (Medina et al, 2011). The global population of domestic cats worldwide is estimated at 600 million, including 74 million pet cats and 60-100 million feral cats in the United States (The Wildlife Society, 2011; 2012 US Pet Ownership and Demographics Source Book; Jessup, 2004). Cats can reach very high population densities as a result of artificial food subsidies, thus, even if individual cats have relatively low predation rates because they do not need to hunt in order to eat, the overall effects of cat predation may be severe (Baker et al, 2005). Cats have caused intense conservation problems on island systems where there are 33 examples of cats being directly responsible for the extirpation of island species, and another 37 cases in which domestic cats had serious impacts resulting in drastic reductions in populations (Medina et al, 2011). This loss or reduction of endemic populations can have alarming impacts on local diversity beyond direct predation pressures. This can vary to reducing the behavioral diversity of potential prey species to disrupting important ecological processes, such as seed dispersal by small vertebrates (Medina et al, 2014). The problem continues today, with cats impacting 120 islands throughout the world, resulting in 175 threatened species suffering negative impacts of island cats (IUCN Redlist, 2008).

The ecological impacts of cats in mainland ecosystems is less clear. Although some estimates suggest that cats kill many billions of birds and mammals each year in the United States (Loss, Will \& Marra, 2012), the distribution of cats appears to be mostly restricted to areas near development rather than large natural areas, where predators like coyotes (Canis latrans) 
78 are less likely to occur (Crooks \& Soule, 1999; Kays et al, 2015). Although there are examples of

79 cats affecting prey populations in the mainland (Loss \& Marra, 2017), there are also examples

80 where no relationship was found (Kays \& Dewan, 2004; Sims et al, 2008; Lilith, Calver \&

81 Garkaklis, 2010). Nonetheless, there is a general consensus that, due to the high densities of cats

82 in urban areas, they still have a high potential to negatively affect native prey populations

83 (Woods, McDonald \& Harris, 2003; Kitts-Morgan, 2015; Baker et al, 2008), and that we should

84 follow a precautionary management strategy (Calver et al, 2011).

Most studies into the ecological impact of cats have used owner reports of what they kill,

86 to estimate predation metrics. These studies have revealed great variation in predation rates,

87 ranging from 4-72 prey/cat/year (Baker et al, 2008; Ruxton, Thomas \& Wright, 2002) and are

88 subject to bias as cats tend to only return about $20-30 \%$ of prey items caught (Kays and Dewan,

89 2004; Lloyd et al, 2013). A further complication is that the prey returned to owners does not

90 necessarily reflect what is eaten and how much is eaten. A study in Poland that looked at both

91 owner reports and more direct methods such as fecal analyses and stomach contents revealed that

92 while cats most frequently returned and consumed rodents, mice were brought back much more

93 often than voles, but consumed much less often (Krauze-Gryz, Gryz, \& Goszczyński, 2012).

94 This same study also showed that using pet owners to record prey brought home results in a

95 drastic underestimate to what was being eaten, highlighting the need for methods other than

96 owner reporting to study diet.

97 Stable isotopes offer an alternative approach to study predator ecology, with $\delta^{13} \mathrm{C}$ values

98 reflecting the original source of the carbon as coming from either $\mathrm{C}_{3}$ or $\mathrm{C}_{4}$ plants and $\delta^{15} \mathrm{~N}$ values

99 typically reflecting trophic level (Ehleringer et al, 2015). Because many pet foods use corn $\left(\mathrm{a} \mathrm{C}_{4}\right.$

100 plant), or corn-fed livestock, as a primary ingredient, cats eating primarily pet food are expected 
101 to exhibit much higher $\delta^{13} \mathrm{C}$ values than those primarily eating native prey in forested areas,

102 which tend to feed on $\mathrm{C}_{3}$ plants. Kays and Feranec (2011) used this approach to classify the

103 range wolves (Canis lupus) in the Northeastern U.S. as likely escaped captives or likely natural

104 immigrants, while Cove et al (2018) used it to estimate the percentage of diet for individual feral

105 cats in south Florida. However, both studies used only a few samples of domestic food, which

106 might not reflect the true variety of foods available for pets.

107 Here we extend the isotopic study of cat diet by focusing on pet cats allowed to spend

108 time inside and outside. By obtaining a sample of the exact food being fed to each cat and

109 comparing these with the isotopic values of their tissue (hair), we expect to have high power to

110 discern the proportion of their diet that comes from pet food and native prey. We also extend

111 sampling of pet food to two countries, the United States (US) and United Kingdom (UK), with

112 different reliance on corn-based food products.

113

114 MATERIALS AND METHODS. Cat food and hair sample collection. ---We recruited

115 volunteer pet owners through blog posts, social media, and through a Citizen Science vending

116 machine located at the Oakland Museum of California. Most participants were from Cornwall,

117 UK (106), North Carolina (37), Long Island, NY and southern Connecticut (18), and Oakland,

118 CA (12). The remainder (29) were scattered around the US. Volunteers were asked to send us a

119 small bag of their cat's hair, one tablespoon of their cat's dry pet food and information about

120 how often the cat goes outside, the brand and flavor of the pet food, and notes about any other

121 kinds of food the cat may be consuming (human food, treats, grass, potential prey, etc.). If the cat

122 consumed wet pet food, we purchased a sample of each brand/flavor specified rather than asking

123 volunteers to send a wet sample through the mail. Volunteers were also asked to specify any 
124 food changes in the six months prior to collection of hair. We recorded the price of each type of

125 cat food from the US from Chewy.com except for store specific brands (Harris Teeter ${ }^{\mathrm{TM}}$ Your

126 Pet, Trader Joe's ${ }^{\mathrm{TM}}$ Cat Food, Kirkland Signature ${ }^{\mathrm{TM}}$, and Paws and Claws Delicious Mix ${ }^{\mathrm{TM}}$ )

127 which were obtained from the websites of those retailers.

128 Our methods and research are approved by the North Carolina State Institutional Review

129 Board (\#3515) and the Animal Care and Use Committee of the North Carolina Museum of

130 Natural Sciences (NCSM 2014-01). All participants filled out an informed consent form prior to

131 providing us with samples and diagnostic information about their cats.

132 Sample preparation. ---All samples of dry food were ground into a uniformly sized

133 powder using a coffee grinder, which was rinsed with water and cleaned with ethyl alcohol

134 between each sample to prevent contamination. Homogenized samples were stored in separate

135 plastic containers and dried at 40 degrees Celsius for a minimum of 24 hours to remove any

136 excess moisture. All wet food was freeze dried and ground into a powder. Small portions of the

137 samples of cat hair provided were treated with a chloroform mixture containing a ratio of

138 1.0:2.0:0.8 parts chloroform, methanol, and water respectively for one hour in a sonic bath, and

139 then rinsed with deionized water before drying the samples at $40^{\circ} \mathrm{C}$ (Kays \& Feranec, 2011).

140 Samples of hair and feathers of potential prey items were obtained from the Bird and Mammal

141 Collections at the North Carolina Museum of Natural Sciences and were treated using the same

142 process as was used for preparing the samples of cat hair.

143 Once cleaned and/or dried, all samples (hair, feathers, dry food, and wet food) were

144 weighed using an electric balance to a precision of $0.1 \mathrm{mg}$ into roughly $2 \mathrm{mg}$ portions (we

145 accepted weights between 1.8 and $2.2 \mathrm{mg}$ ) and placed into $5 \times 9 \mathrm{~mm}$ tin capsules. Each tin capsule

146 was folded into a cube and placed into one of three 96 well plastic sample trays. Sample trays 
147 from US were sent to the Center for Stable Isotope Biogeochemistry at the University of

148 California at Berkeley for analysis while samples from the UK were sent to Elemtex Stable

149 Isotope Analysis. Samples at each location underwent the same process to determine isotopic

150 composition. Isotopes were reported to a long-term precision of $0.1 \%$ for $\delta^{13} \mathrm{C}$ and $0.2 \%$ for $151 \delta^{15} \mathrm{~N}$.

152 Analysis. --- Cats from the southeastern US were run through a Bayesian two-tracer

153 mixing model using the MixSIAR package in R (Stock \& Semmens, 2016), which uses a

154 Markov Chain Monte Carlo (MCMC) simulation to estimate the components of each individual

155 cat's diet by modeling the proportions of its sources. Only these cats were considered as all

156 reliable prey isotope values obtained were from this region. To simplify the model, all native

157 prey was averaged into one source and compared against the different types of pet food each cat

158 was known to eat. All models were run with the normal setting of MCMC in the MixSIAR GUI,

159 which has a chain length of 100,000 on three chains. The MixSIAR model also incorporates

160 uncertainty due to the discrimination factor between consumers and sources into the model.

161 Because there are no species-specific discrimination factors calculated for domestic cats to

162 account for changes in isotope values as food sources are incorporated into cat tissue, we created

163 our own value using the diet of one indoor-only cat which ate only one type of cat food. We used

164 the difference in the isotopic value between that cat's food and hair $\left(+1.9 \%\right.$ for $\delta^{15} \mathrm{~N}$ and +2.6

$165 \%$ for $\delta^{13} \mathrm{C}$ ) as a discrimination factor for the rest of our cats, which was lower in adjustment for

$166 \delta^{15} \mathrm{~N}$ compared to the typical value used by carnivore studies $\left(+3.2 \%\right.$ for $\delta^{15} \mathrm{~N}$ and $+2.6 \%$ for

$167 \delta^{13} \mathrm{C}$ ), derived from red fox (Roth \& Hobson 2000). 
Because the mixing models require all food sources to have $\mathrm{n}>1$ for the complete model

169 to run, we combined foods made by the same manufacturer into brand averages, keeping dry

170 food, fish based wet food, and meat based wet food in separate categories.

171 Results were compared statistically using Geweke and Gelman-Rubin diagnostics to

172 verify models and then using percent credible intervals (posterior probabilities) as a primary

173 basis of determining relative contributions of different items to the diet of each cat. Isospace

174 plots and scaled posterior density charts were used as a visual aid to this process. Cats were then

175 categorized into those that likely consumed prey, those that likely ate only cat food, those that

176 had an uncertain diet, and those that isotope values outside range of food sources. This type of

177 analysis works regionally in areas where the vegetation relies on mostly $\mathrm{C}_{3}$ photosynthesis, and

178 pet food is mostly composed from ingredients that come from $\mathrm{C}_{4}$ plants. This results in native

179 plants and animals having lower $\delta^{13} \mathrm{C}$ values than those in pet food.

180 Cat classification. ---Cats were categorized into one of four categories after being run

181 through the MixSIAR mixing model based on the median posterior probabilities generated by the

182 MixSIAR model and isospace plots. The categories were as follows: cats that likely ate some

183 prey, labelled as Hunters; cats that likely eat only cat food, labelled as Non-hunters; cats that had

184 an uncertain diet, labelled as Uncertain; and cats that had high carbon isotope values outside

185 reasonable ranges from food sources, labelled as High-C. Because the mixing models cannot run

186 with a food source represented by a single sample, we used an average of potential prey values

187 (CombinedPrey) and also averaged individual food samples from the same brand. Some brands

188 of food still only had one sample, and thus could be evaluated with isospace plots but not mixing

189 models. Cats were considered to be Hunters if the median proportion value, the $50 \%$ credible

190 interval of the posterior probabilities generated through the MixSIAR model, was greater than or 
191 equal to 0.245 and the isospace plot showed the cat as being distinguishably closer to the prey

192 source than the cat food source(s). Cats were labelled as Non-hunters if the median proportion

193 value was less than 0.245 with a relatively small amount of variation in the $95 \%$ confidence

194 interval generated from the $2.5 \%$ and $97.5 \%$ credible intervals generated by the MixSIAR model

195 and the isospace plot showed the cat as being distinguishably closer to the cat food source(s).

196 Cats were labelled as High-C when the isospace plot showed the cat as having a more positive

197 carbon value that was outside the range of variation for all potential dietary sources.

198 Because the MixSIAR model constructs a proposed diet only for the sources input into

199 the model, the posterior probability values were not considered for cats that fit the High-C

200 category, as an unknown source affecting their diet was not included in the model. Cats were

201 labelled as Uncertain when the median proportion value was less than 0.245 but there was a large

202 amount of variation shown by the $95 \%$ confidence interval generated by the $2.5 \%$ and $97.5 \%$

203 credible intervals and were roughly equidistant from all potential dietary sources on the isospace 204 plot.

205

206

RESULTS. Isotope values. ---We obtained isotope values for 47 samples from 13

species of potential prey, while 6 prey samples did not run correctly: 2 samples returned no data and 4 returned extraneous values that did not fit the data (Table S1). Values of $\delta^{13} \mathrm{C}$ and $\delta^{15} \mathrm{~N}$ for all potential native prey from the southeastern US ranged from $-17.7 \%$ to $-25.3 \%$ and $+2.0 \%$ o to $+9.5 \%$ respectively and averaged $-21.9 \%( \pm 2.1 \mathrm{SD})$ for $\delta^{13} \mathrm{C}$ and $+5.5 \%$ ( $\left.\pm 1.7 \mathrm{SD}\right)$ for

$211 \delta^{15} \mathrm{~N}$. Individuals of the same prey species tended to have similar overall isotope values. Only 7

212 prey samples had $\delta^{13} \mathrm{C}$ values higher than -20\%, including all Mus musculus samples, 1 Pipilo

213 erythrophthalmus, 1 Cardinalis cardinalis, and 1 Zenaida macroura. 

samples of wet cat food from 27 different brands representing 55 different flavors of cat food

216 (Table S1). Dry cat food values of $\delta^{13} \mathrm{C}$ and $\delta^{15} \mathrm{~N}$ averaged $-19.3 \%$ o $( \pm 2.3 \mathrm{SD})$ and $+4.2 \%$ o $( \pm 1.3$

$217 \mathrm{SD})$ respectively while wet food values averaged $-18.9 \%$ ( $\pm 1.6 \mathrm{SD})$ and $+5.9 \%$ ( $\pm 3.0 \mathrm{SD})$ and nitrogen values nearly $15 \%$ (Fig. 1; Fig. S1). The carbon variation was relatively uniform. Nitrogen variation included one outlier from a tuna-based food, with the next highest value 7\%o lower. Two-tailed T-Tests revealed that $\delta^{13} \mathrm{C}$ values differed significantly between potential prey and dry food $(\mathrm{p}<0.001)$ as well as wet food $(\mathrm{p}<0.001)$. Values for $\delta^{15} \mathrm{~N}$ differed significantly between potential prey and dry food $(p=0.007)$ but did not differ significantly between wet food 224 and prey.

The 112 food samples from the UK consisted of 61 samples of dry food and 51 samples of wet food. These samples exhibited wide variation with carbon values ranging over $11 \%$ and nitrogen values ranging nearly $13 \%$ (Table S2). Food from the UK was also distinctly lower in carbon compared to food from the US (Fig. 1; Fig. S2). We had multiple samples for 7 US brands, and for 15 US flavors. We found high variation within a brand, with $\delta^{13} \mathrm{C}$ varying as much as $6.1 \%$, and $\delta^{15} \mathrm{~N}$ as much as $6.3 \%$ within a brand (excluding the outlier from tuna food,

231 Table S1, Fig. 2A).

We included isotope values for 96 individual cats from the US of which 47 were female and 49 were male. Cats averaged 8.7 years old $( \pm 3.7 \mathrm{SD})$, but ranged from 1 to 17 years. Isotope 234 values of $\delta^{13} \mathrm{C}$ and $\delta^{15} \mathrm{~N}$ for cats averaged $-16.9 \%( \pm 1.6 \mathrm{SD})$ and $+6.8 \%$ ( $\left.\pm 0.9 \mathrm{SD}\right)$ respectively. 235 Cats also had a fair amount of variation, carbon values ranged about $6.1 \%$ and nitrogen values 236 ranged about $5.8 \%$. 
238 and $+6.7 \%$ o $( \pm 0.7 \mathrm{SD})$ respectively. Carbon values for cats varied a fair amount ranging about 6

$239 \%$ while nitrogen values only ranged about $3 \%$. Age and sex data were not included for the cats

240 from the UK. Two-tailed T-tests showed that carbon values differed significantly between the

241 US and UK for cats $(p<0.001)$, dry food $(p<0.001)$, and wet food $(p<0.001)$, while nitrogen

242 values only differed significantly for wet food $(\mathrm{p}=0.017)$.

243 Classifying cats. ---Of the 47 cats from the southeastern US that we could compare to

244 regional prey 38 cats were successfully run through the MixSIAR model, while 9 were unable to

245 be run due to having food sources with only one value. All cats successfully run through the

246 model met the criteria of the Geweke and Gelman-Rubin tests. There were 13 cats classified as

247 hunters (Fig. 3A), 7 cats classified as non-hunters (Fig. 3B), and 11 cats classified as high carbon

248 (Fig. 3C). All but one of the 11 cats classified as high carbon would have been classified as

249 hunters based on posterior probabilities in MixSIAR. The remaining 16 cats were unable to be

250 accurately classified based on the mixing models due to intermediate posterior probability values

251 and high levels of variation and were labeled as uncertain (Fig. 3D). Plotting cats by assigned

252 category in an isospace plot of all possible pet food showed extensive overlap between categories 253 (Fig. 4).

254 Average isotope values for both cats and food differed a bit between categories. Mean cat $255 \delta^{13} \mathrm{C}$ values were significantly different in hunters (-16.99 \%o $\left.\pm 1.8 \mathrm{SD}\right)$ and High-C cats $(-16.55$ $256 \% \pm 0.9 \mathrm{SD})$ than those in non-hunters $(-15.65 \% \pm 0.5 \mathrm{SD}, \mathrm{p}=0.02$ and $\mathrm{p}=0.01$ respectively $)$. The 257 mean cat $\delta^{13} \mathrm{C}$ values of hunters and High-C cats did not differ significantly $(\mathrm{p}=0.44)$. There were 258 no significant differences in mean cat $\delta^{15} \mathrm{~N}$ values between all categories; all categories had 259 means between +6.52 and $+6.88 \% \delta^{15} \mathrm{~N}$. The mean food $\delta^{13} \mathrm{C}$ value of High-C cats $(-20.57 \%$ 
$260 \pm 1.4 \mathrm{SD})$ was significantly different than that of hunters $(-18.10 \% \pm 2.2 \mathrm{SD}, \mathrm{p}=0.003)$ and non-

261 hunters $(-19.06 \% \pm 0.9 \mathrm{SD}, \mathrm{p}=0.014)$. There was no significant difference in the mean food $\delta^{13} \mathrm{C}$

262 values of hunters and non-hunters ( $\mathrm{p}=0.18)$. Mean food $\delta^{15} \mathrm{~N}$ values were significantly different

$263(\mathrm{p}=0.007)$ between non-hunters $(+5.49 \% \pm 1.1 \mathrm{SD})$ and High-C cats $(+3.88 \% \pm 0.5 \mathrm{SD})$. The

264 mean food $\delta^{15} \mathrm{~N}$ value for hunters $(+4.45 \% \pm 1.2 \mathrm{SD})$ did not differ significantly from non-

265 hunters and High-C cats ( $\mathrm{p}=0.076$ and $\mathrm{p}=0.155$ respectively).

266 A two-tailed T-Test revealed sex did not account for any difference in the likelihood of a

267 cat being assigned to a category $(\mathrm{p}=0.63)$. Cats ranged between 1 and 17 years old. Age had little

268 impact on the likelihood of cats being assigned to any category; there was a very weak

269 correlation $\left(\mathrm{R}^{2}=0.0093\right)$ between age and category.

270 Prices for US pet foods ranged from $\$ 1.24$ per kilogram to nearly $\$ 25$ per kilogram. Food

271 price per unit was significantly negatively correlated with carbon values $\left(R^{2}=0.108, p=0.00018\right)$,

272 but had no relationship with nitrogen values $\left(\mathrm{R}^{2}=0.0144, \mathrm{p}=0.18\right)$ (Fig. 2B). Comparing the

273 average food price per kilogram between the assigned categories of cats using a two tailed T-test

274 revealed no significant difference between hunters $(\$ 3.87 / \mathrm{kg} \pm \$ 2.90 \mathrm{SD})$ and non-hunters

$275(\$ 3.16 / \mathrm{kg} \pm \$ 1.00 \mathrm{SD}, \mathrm{p}=0.43)$, and no significant difference between hunters and cats classified

276 as High-C $(\$ 5.44 / \mathrm{kg} \pm \$ 2.77 \mathrm{SD}, \mathrm{p}=0.19)$. There was a significant difference between non-

277 hunters and High-C cats $(\mathrm{p}=0.03)$.

278 Cats grouped in the uncertain category also had several significant differences from cats

279 in other categories. The mean cat $\delta^{13} \mathrm{C}$ value of uncertain cats $(-17.83 \% \pm 1.4 \mathrm{SD})$ was

280 significantly different from non-hunters $(\mathrm{p}<0.001)$ and High-C cats $(\mathrm{p}=0.007)$. The mean food

$281 \delta^{13} \mathrm{C}$ value of uncertain cats $(-19.66 \% \pm 0.9 \mathrm{SD})$ was significantly different from hunters

282 ( $\mathrm{p}=0.028)$. The mean food $\delta^{15} \mathrm{~N}$ value of uncertain cats $(+6.22 \% \pm 1.6 \mathrm{SD})$ was significantly 
283 different from hunters $(\mathrm{p}=0.002)$ and High-C cats $(\mathrm{p}<0.001)$. The average food price per

284 kilogram of uncertain cats $(\$ 4.97 / \mathrm{kg} \pm \$ 1.95 \mathrm{SD})$ was significantly different from non-hunters $285(\mathrm{p}=0.006)$.

DISCUSSION. Because most pet cats are consistently fed one kind of food at home, and

because many pet foods are thought to include corn or corn-fed livestock (Schnepf, 2011; Galera et al, 2019), we expected that a stable isotope approach would have a high power to discern pets consuming native prey from those eating only pet food. However, we found that the high variability of isotope values for pet foods, across and within brands, not to mention across countries, makes it extremely difficult to determine the diet of individual cats. By limiting the potential cat foods for each individual to what their owners fed them, we were able to use mixing models to classify $28 \%$ of cats we sampled in the southeastern US as likely hunters, $15 \%$ as nonhunters, while $34 \%$ were unknown and $23 \%$ had unexplainably high C values (Fig. 3). However, if we did not have information from owners about the brand of food they use, for example, as would be the case for feral cats, it would be impossible to categorize the diets of cats with any confidence based on isotope values alone (Fig. 4). It is also important to consider the fact that cats frequently only consume a portion of the prey they kill, potentially leaving only very small influences on the overall diet of an animal. Therefore, counter to our expectations, we found that isotope analysis of cat hair has little value in determining if a cat has been eating wild prey as a

302 result of both high variability in the carbon and nitrogen values of pet foods, the hunting habits 303 of cats.

The high level of variation between different cat food, including many of the flavors 
306 samples of cat food directly from owners and thus only had broad descriptions of the flavors

307 from the volunteers who sent them. Having access to the labels with the nutrition information

308 and ingredients may have helped to understand the variability between foods, however many pet

309 food labels lack specific information about the main sources of carbohydrates, protein, and fat

310 provided and can be misleading (Galera et al, 2019). Among dry food alone there was a range of

311 nearly $10 \%$ in $\delta^{13} \mathrm{C}$ values. In comparison, all species of our native prey combined had $\delta^{13} \mathrm{C}$

312 values ranging $7.6 \%$. Though $\delta^{13} \mathrm{C}$ values in both wet food and dry food were significantly

313 different from the values of potential prey, the variation within cat food $\delta^{13} \mathrm{C}$ values still

314 encompassed a large amount of the variation in the values of potential prey, resulting in

315 difficulty in isolating food sources (see Fig.S1). The largest amount of variation in $\delta^{13} \mathrm{C}$ values

316 from a single mammalian prey source was $3.54 \%$ from the woodland vole, while for a single

317 bird prey source it was $6.21 \%$ from the eastern towhee. It is also important to consider that

318 many of the animals selected as potential prey have diets consisting of a variety of different

319 plants and/or invertebrates. In an animal that eats only one or a select few food sources, such as a

320 grazing Merino sheep, the $\delta^{13} \mathrm{C}$ value range is $0.84 \%$ (Männel, Auerswald \& Schnyder, 2007).

321 The ranges in food isotope values we obtained are very similar to those obtained in a recent

322 Brazilian study looking at the contents of food for domestic dogs (Galera et al, 2019). The cats in

323 our study, from both the US and UK, had $\delta^{13} \mathrm{C}$ value ranges of around $6 \%$. Cats classified as

324 hunters also had a similar range. Considering that many of these animals are supposedly only

325 consuming pet food, we would expect to see little variation in cats, especially those classified as

326 non-hunters. However, the variation from different types of pet food makes it quite difficult to

327 differentiate dietary inputs. The samples received from participants and the wet food purchased

328 from stores in the Raleigh, NC area made up a total of 27 brands, and 55 individual flavors of 
329 food. Purina ${ }^{\mathrm{TM}}$ was the most frequent brand of cat food received and was made up of 14 different

330 flavors of cat food. Some isotopic variation between flavors would be expected here, but

331 surprisingly, $\delta^{13} \mathrm{C}$ values varied more than $5 \%$ across Purina ${ }^{\mathrm{TM}}$ dry foods. Even foods within the

332 same flavor could be variable, for example, samples of Meow Mix ${ }^{\mathrm{TM}}$ Original Choice flavor

333 varied $2 \%$ in $\delta^{13} \mathrm{C}$ values. This suggests that large scale pet food manufacturers vary the

334 ingredients over time. The only clear relationship with the isotope values we obtained from pet

335 food from the US was that the least expensive foods tended to have higher $\delta^{13} \mathrm{C}$ values, with

336 most foods priced less than a dollar per kilogram forming a cluster distinct from other foods on

337 an isospace chart at around - $16 \%$ (Fig. 2B). This may be the result of the incorporation of more

338 corn and corn byproducts into inexpensive foods.

339 Cat foods from the United Kingdom ranged over a similar level of variation as those in

340 the US, but their carbon values were noticeably lower (Figure S2). The difference in C can likely

341 be explained by the fact that less corn is used in pet foods within the UK (Howsam, 2018).

342 Statistics on corn use for animal feed in both countries shows that nearly 700 times as many

343 tonnes of corn is used in the US, and that corn makes up a much larger proportion of the

344 ingredients in animal feed in the US as well (Schnepf, 2011). Wheat and barley are the primary

345 grain ingredients in animal feed in the UK (Howsam, 2018), both of which use the $\mathrm{C}_{3}$

346 photosynthetic pathway, again contributing to the lower $\delta^{13} \mathrm{C}$ values in this food. This difference

347 brings into question the extent that high $\mathrm{C}$ isotopic values can be used as a global indication of

348 domestic foods in the diet of wild animals (Penick, Savage \& Dunn, 2015), however, we do not

349 mean to say that using isotope analysis is not both useful and very appropriate in other situations

350 as it has been proven that the use of stable isotopes can aid in answering complex diet and food

351 web problems (Post, 2002). 
By restricting our comparisons to only the cat food brands eaten by an individual cat, our

353

models classified 13 as hunters, 7 as non-hunters, and 16 as having an uncertain diet. This high

level of uncertainty leaves us unable to offer broader generalizations as to the true proportion of cats in our study that consumed native prey. Both hunters and non-hunters were fed less expensive food on average than were cats classified as "uncertain." Since less expensive foods tended to be higher in $\delta^{13} \mathrm{C}$ values, these foods had values more different than those of native prey, giving them a greater potential to be categorized as either hunters or non-hunters due to a wider gap between sources. However, the overall average price per kilogram of food only differed significantly between non-hunters and uncertain cats. This is probably because the higher $\delta^{13} \mathrm{C}$ values of inexpensive foods allowed better discrimination against the low $\delta^{13} \mathrm{C}$ values typical of most native prey. However, in the case of hunters, an alternative explanation could be that poor nutrition associated with less expensive food motivates cats to supplement their diet with more native prey. Indeed, cats given a choice of multiple pet foods will select foods to balance their macronutrients (Hewson-Hughes et al, 2012). This supports the possibility that cats with access to only one pet food, especially an inexpensive grain-based food, might be more likely to hunt to supplement their diet. This is especially interesting considering that less expensive food is likely what is given to managed feral cat colonies and could potentially be resulting in negative consequences. Testing this hypothesis would require non-isotope diet data to avoid confounding effects of less expensive food offering better isotopic discrimination, due to its typically higher $\delta^{13} \mathrm{C}$ values.

The other 11 cats that were categorized as having a high carbon diet had carbon isotope values that were more positive than any potential food sources, both wildlife and cat food. The average $\delta^{13} \mathrm{C}$ values of these cats were very similar to those of Hunters, however, the food they 
375 were given was more expensive than cats classified as hunters, and significantly more so than

376 non-hunters (Figure 2B). This more expensive food also tended to have slightly lower $\delta^{13} \mathrm{C}$

377 values making the cats visually appear as though they had higher $\delta^{13} \mathrm{C}$ values. It is possible these

378 cats are being supplemented with less expensive cat foods, or potentially table scraps, in addition

379 to what was reported, contributing to $\delta^{13} \mathrm{C}$ values higher than that of the food they normally eat.

380 Cats were ancestrally obligate carnivores. In contrast to dogs, little evidence exists for

381 omnivory among felids in general or the wild relatives of domestic cats. By 7500 BCE cats show

382 some evidence of domestication (Ottoni et al, 2016), and by 5300 BCE some populations of cats

383 became partially dependent on food sources associated with human settlements (Hu et al, 2014).

384 However, while dogs were able to eat a variety of foods, including some plant matter, early

385 domestic cats appear to have been primarily preying on other species that were eating grain,

386 rather than grain or other plant matter itself. As a result, while dogs evolved a number of

387 adaptations for feeding on starches during domestication, including multiple copies of amylase

388 genes (Axelsson et al, 2013), the same does not appear to be the case for cats (Di Cerbo et al,

389 2017). In this light, it is interesting to consider the modern diets of cats. This diet appears to be

390 different from their ancestral diet in several ways. First, cats now consume different prey species

391 than they might have historically. For example, tuna and other large fish are present in many

392 varieties of cat food. Second, at least some cats have diets in which grains feature prominently.

393 Third, and perhaps most generally, cats now have diets that vary greatly both because of where

394 cats live and because of the fluctuating composition of cat foods. It will be interesting to consider

395 the ways in which this change might be expected to affect the gut microbes, nutrition, health and

396 well-being of cats. 
Any isotope ecology study needs to consider the trophic enrichment/discrimination

factor, which describes the change in isotope values as food is incorporated into the tissue of an animal. This factor varies across species (Caut, Angulo \& Courchamp, 2009), and most studies of carnivores have used the factor $+3.2 \%$ for $\delta^{15} \mathrm{~N}$ and $+2.6 \%$ for $\delta^{13} \mathrm{C}$ derived for red foxes by Roth and Hobson (2000). Preliminary analyses of our data suggested that this adjustment value might not represent cats well, as also noted by other feline studies (Newsome et al, 2015; Parng,

403 Crumpacker \& Kurle, 2014). Therefore, we took advantage of one of our study animals that was

404 a non-hunting indoor only cat fed only one type of dry cat food to derive a cat specific

405 discrimination factor of $+1.9 \%$ for $\delta^{15} \mathrm{~N}$ and $+2.6 \%$ for $\delta^{13} \mathrm{C}$. This is identical to the $\mathrm{C}$

406 adjustment as found by Roth and Hobson (2000) but lower than their N adjustment. Using this

407 value improved the fit of the models in our study, resulting in many more cats appearing in the

408 bounds of reasonable variation between food sources and isospace plots. However, we recognize

409 that this post-hoc analysis of one animal is not a replacement for a controlled experiment with

410 multiple individuals and suggest that future studies should consider adding these to their protocol

411 to improve the discrimination factor estimation for cats. In addition to the need to combine

412 individual food samples into brand averages and the approximated trophic enrichment factor, this

413 study faced several other limitations. A relatively high degree of overlap between the isotope

414 values of potential prey sources and pet food sources limited our ability to identify clear

415 distinctions between the two. Our comparison of native prey was restricted to cats living in the

416 southeastern US, where we had adequate sampling of potential bird and mammal prey, although

417 no values for arthropods or herpetofauna, which occasionally are killed by cats (Woods,

418 McDonald \& Harris, 2003; van Heezik et al, 2010; Doherty et al, 2015). Finally, domestic cats

419 have been proven to not always eat what they kill when hunting. Research shows that often 
420 nearly $50 \%$ of the kills of outdoor owned cats are left at the site (Lloyd et al, 2013). This limits

421 the effectiveness of using diet alone to identify the impacts on local wildlife.

422

CONCLUSIONS. Stable isotope analysis is a useful tool in identifying broad dietary

423 patterns across trophic levels (from N) and the origin of plant material (from C). However, due to

424 the surprisingly high level of variation found in isotopic values across different pet foods, we

425 conclude that it is very difficult to distinguish the diet of a cat based on its isotopic values, and

426 thus of little value for future studies of cats predation on native species. Indeed, even though we

427 knew the brand of cat food eaten by an individual cat we were still not able to classify them as a

428 hunter or non-hunter in $57 \%$ of the cases; this would be considerably more difficult in the case of

429 feral or free-ranging cats where the exact brand of pet food is unknown or variable. Furthermore,

430 even perfect isotope studies would be unable to account for the prey killed by cats but not eaten.

431 At least $28 \%$ of the cats in our study showed evidence of having consumed wild prey, while this

432 is probably an underestimate, it confirms the risk cats can pose to native prey and the importance

433 of studying the phenomenon more. To truly understand the impacts cats have on the wildlife we

434 agree with Krauze-Gryz et al (2012) that a diversity of approaches will be needed since "Not

435 everything is brought home, certain species are eaten more preferentially to others, and small

436 prey may go unnoticed."

437

438

AKNOWLEDGEMENTS. We would like to thank the volunteers who participated in

this, and other Cat Tracker studies, Tim Walsh for assisting with Cat Tracker volunteers through

440 the Bruce Museum in Connecticut, and Lea Shell and Neil McCoy at North Carolina State

441 University for their help in organizing the Cat Tracker email and website through Your Wildlife. 


\section{REFERENCES}

444 American Veterinary Medical Association,. (2012). U.S. Pet Ownership \& Demographics $445 \quad$ Sourcebook.

446 Axelsson, E., A. Ratnakumar, M. L. Arendt, K. Maqbool, K. T. Webster, M. Perloski, O. Liberg, 447 J. M. Arnemo, Å. Hedhammar, and K. Lindblad-Toh. 2013. The genomic signature of dog 448 domestication reveals adaptation to a starch-rich diet. Nature 495(7441): 360-364.

449 Baker, P. J., A. J. Bentley, R. J. Ansell, and S. Harris. 2005. Impact of predation by domestic $450 \quad$ cats Felis catus in an urban area. Mammal Review 35(3-4): 302-312.

451 Baker, P. J., S. E. Molony, E. Stone, I. C. Cuthill, and S. Harris. 2008. Cats about town: Is 452 predation by free-ranging pet cats Felis catus likely to affect urban bird populations? Ibis 453 150(SUPPL.1): 86-99.

454 Calver, M.C., J. Grayson, M. Lilith, and C.R. Dickman. 2011. Applying the precautionary 455 principle to the issue of impacts by pet cats on urban wildlife. Biological Conservation 144: $456 \quad 1895-1901$.

457 Caut, S., E. Angulo, and F. Courchamp. 2009. Variation in discrimination factors $(\Delta 15 \mathrm{~N}$ and $458 \Delta 13 \mathrm{C})$ : The effect of diet isotopic values and applications for diet reconstruction. Journal of $459 \quad$ Applied Ecology 46(2): 443-453.

460 Cove, M. V., B. Gardner, T. R. Simons, R. Kays, and A. F. O’Connell. 2018. Free-ranging 461 domestic cats (Felis catus) on public lands: estimating density, activity, and diet in the 462 Florida Keys. Biological Invasions 20(2): 333-344.

463 Crooks, D.R. \& M.E. Soule. 1999. Mesopredator release and avifaunal extinctions in a 464 fragmented system. Nature 400: 563-566.

465 Di Cerbo, A., J.C. Morales-Medina, B. Palmieri, F. Pezzuto, R. Cocco, G. Flores, and T. Ianitti. 

Science 112: 161-166.

468

469

470

471

472

473

474

475

476

477

478

479

480

481

482

483

484

485

486

487

488

Doherty, T. S., R. A.Davis, E. J. B. van Etten, D. Algar, N. Collier, C. R. Dickman, and S. Robinson. 2015. A continental-scale analysis of feral cat diet in Australia. Journal of Biogeography 42(5): 964-975.

Ehleringer, J.R., L.A. Chesson, L.O. Valenzuela, B.J. Tipple, and L.A. Martinelli. 2015. Stable isotopes trace the truth: from adulterated foods to crime scenes. Elements 11(4): 259-264.

Galera, L.dA., A.L. Abdalla Filho, L.S. Reis, J.L. de Souza, Y.A. Hernandez, and L.A. Matinelli. 2019. Carbon and nitrogen isotopic composition of commerical dog food in Brazil. PeerJ $7: \mathrm{e} 5828$

Hewson-Hughes, A.K., V.L. Hewson-Hughes, A. Colyer, A.T. Miller, S.R. Hall, D. Raubenheimer, and S.J. Simpson. 2012 Consistent proportional macronutrient intake selected by adult domestic cats (Felis catus) despite variations in macronutrient and moisture content of foods offered. Journal of Comparitive Physiology B 183(4): 525-536.

Howsam, A. (2018) Animal Feed Statistics for Great Britain- December 2017. National Statisitics- Department of the Environment, Food, \& Rural Affairs.

Hu, Y., S. Hu, W. Wang, X. Wu, F.B. Marshall, X. Chen, L. Hou, and C. Wang. 2014. Earliest evidence for the commensal processes of cat domestication. Proceedings of the National Academy of Sciences 111(1): 116-120.

IUCN (2008) 2008 IUCN Red List of Threatened Species. IUCN.

Jessup, D. A. 2004. The welfare of feral cats and wildlife. Journal of the American Veterinary Medical Association 225(9): 1377-1383.

JMP®, Version 13 Pro. SAS Institute Inc., Cary, NC, 1989-2007. 
489 Kays, R., R. Costello, T. Forrester, M. Baker, A. Parsons, L. Kalies, G. Hess, J.J. Millspaugh, $490 \quad$ and W. McShea. 2015. Cats are rare where coyotes roam. Journal of Mammalogy 96: 981491987.

492 Kays, R.. and R. S. Feranec. 2011. Using Stable Carbon Isotopes to Distinguish Wild from 493 Captive Wolves. Northeastern Naturalist 18(3): 253-264.

494 Kays, R. W. and A. A. Dewan. 2004. Ecological impact of inside/outside house cats around a 495 suburban nature preserve. Animal Conservation 7: 1-11.

496 Kitts-Morgan, S. E. 2015. Companion animals symposium: Sustainable ecosystems: Domestic 497 cats and their effect on wildlife populations. Journal of Animal Science 93(3): 848-859.

Krauze-Gryz, D., J. Gryz, and J. Goszczyński. 2012. Predation by domestic cats in rural areas of central Poland: An assessment based on two methods. Journal of Zoology 288(4): 260-266.

Lilith, M., M. Calver, and M. Garkaklis. 2010. Do cat restrictions lead to increased species diversity or abundance of small and medium-sized mammals in remnant urban bushland?

\section{PACIFIC CONSERVATION BIOLOGY 16: 162-172.}

Loss, S.R. \& P.P. Marra. 2017. Population impacts of free-ranging domestic cats on mainland

vertebrates. Frontiers in Ecology and the Environment 15: 502-509.

Loss, S. R., T. Will, and P. P. Marra. 2013. The impact of free-ranging domestic cats on wildlife of the United States. Nature Communications 4: 1396.

Loyd, K. A. T., S. M. Hernandez, J. P. Carroll, K. J. Abernathy, and G. J. Marshall. 2013. Quantifying free-roaming domestic cat predation using animal-borne video cameras. Biological Conservation 160: 183-189.

Männel, T. T., K. Auerswald, and H. Schnyder. 2007. Altitudinal gradients of grassland carbon and nitrogen isotope composition are recorded in the hair of grazers. Global Ecology and 
$512 \quad$ Biogeography 16(5): 583-592.

513 Medina, F. M., E. Bonnaud, E. Vidal, B. R. Tershy, E. S. Zavaleta, C. Josh Donlan, and M.

514 Nogales. 2011. A global review of the impacts of invasive cats on island endangered

$515 \quad$ vertebrates. Global Change Biology 17(11): 3503-3510.

516 Medina, F., E. Bonnaud, E. Vidal, and M. Nogales. 2014. Underlying impacts of invasive cats

517 on islands: not only a question of predation. Biodiversity and Conservation 23(2): 327-342.

518 Newsome, S. D., H. M. Garbe, E. C. Wilson, and S. D. Gehrt. 2015. Individual variation in

519 anthropogenic resource use in an urban carnivore. Oecologia 178(1): 115-128.

520 Ottoni, C., W. van Neer, B. De Cupere, J. Daligault, S. Guimaraes, J. Peters, N. Spassov, M. E.

521 Pendergast, N. E. Boivin, A. Morales-Muñiz, A. Bălăşescu, C. Becker, N. Benecke, A.

522 Boronenant, H. Buitenhuis, J. Chahoud, A. Crowther, L. A. Llorente, N. Manaseryan, H.

523 Monchot, V. Onar, M. Osypińska, O. Putelat, J. Studer, U. Wierer, R. Decorte, T. Grange,

524 and E. Geigl. (2016). Of cats and men: the paleogenetic history of the dispersal of cats in

525 the ancient world. bioRxIV (Pre-Print).

526 Parng, E., A. Crumpacker, and C. M. Kurle. 2014. Variation in the stable carbon and nitrogen

527 isotope discrimination factors from diet to fur in four felid species held on different diets.

528 Journal of Mammalogy 95(1): 151-159.

529 Penick, C. A., A. M. Savage, and R.R. Dunn. 2015. Stable isotopes reveal links between human

530 food inputs and urban ant diets. Proceedings of the Royal Society B 282(1806).

531 Post, D.M. 2002. Using stable isotopes to estimate trophic position: Models, methods, and

$532 \quad$ assumptions. Ecology 83(3): 703-718.

533 R Core Team (2013). R: A language and environment for statistical computing. R Foundation for 534 Statistical Computing, Vienna, Austria. URL http://www.R-project.org/. 
535 Roth, J. D., and K. A. Hobson. 2000. Stable carbon and nitrogen isotopic fractionation between

536 diet and tissue of captive red fox: implications for dietary reconstruction. Canadian Journal 537 of Zoology 78(5): 848-852.

538 Ruxton, G. D., S. Thomas, and J. W. Wright. 2006. Bells reduce predation of wildlife by 539 domestic cats (Felis catus). Journal of Zoology 256(1): 81-83.

540 Schnepf, R. (2011). U.S. Livestock and Poultry Feed Use and Availability: Background and $541 \quad$ Emerging Issues. Congressional Research Service, 1-27. R41956

542 Sims, V., K.L. Evans, S.E. Newson, J.A. Tratalos, and K.J. Gaston. 2008. Avian assemblage

543 structure and domestic cat densities in urban environments. Diversity and Distributions 14: $544 \quad 387-399$.

545 Stock, B. C., and Semmens, B. X. (2016). MixSIAR GUI User Manual. 3.1.

546 https://github.com/brianstock/MixSIAR.<doi:10.5281/zenodo.1209993>

547 The Wildlife Society (2011) Feral and free-ranging domestic cats. Final Position Statement

548 van Heezik, Y., A. Smyth, A. Adams, and J. Gordon. 2010. Do domestic cats impose an

549 unsustainable harvest on urban bird populations? Biological Conservation 143(1): 121-130.

550 Woods, M., R. A. McDonald, and S. Harris. 2003. Predation of wildlife by domestic cats Felis $551 \quad$ catus in Great Britain. Mammal Review 33: 174-188. 


\section{Figure 1}

Average isotope values with standard deviations of cats and food sources from the U.S. and U.K.

Food and prey values are adjusted by a trophic enrichment factor of $+2.6 \% \delta 13 \mathrm{C}$ and $+1.9 \% \circ 15 \mathrm{~N}$. Carbon values are more negative for both food and cats in the UK. Higher $813 \mathrm{C}$ values in US cats and food are probably the result of the incorporation of more corn (a C4 photosynthesizing plant) products into cat food, either directly, or through corn-fed livestock. Large variation in $\delta 15 \mathrm{~N}$ values for wet food is probably the result of having ocean fish such as tuna, which feed at a higher trophic level and therefore have higher $\mathrm{N}$ isotopes. 


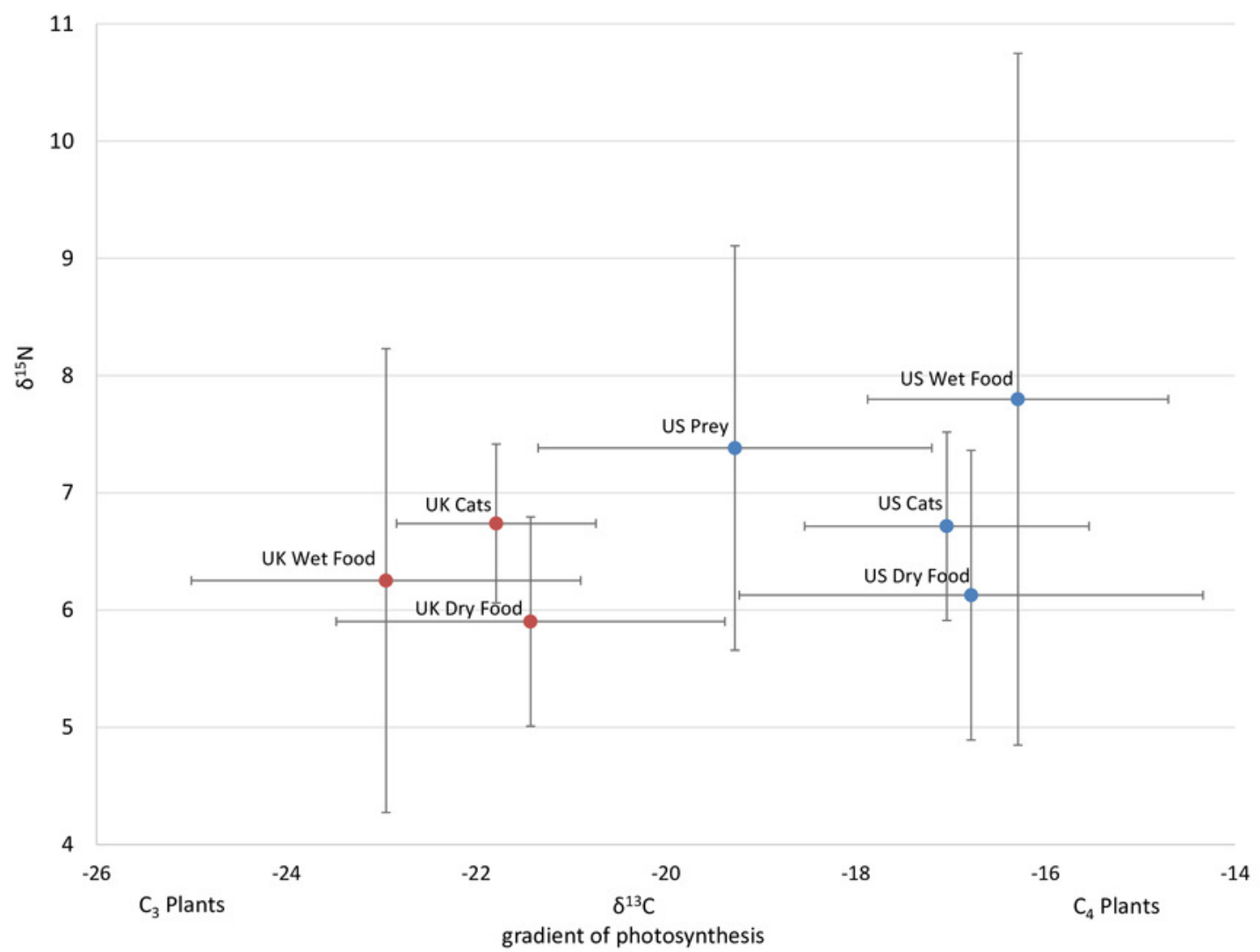


Figure 2

Variation in isotopic values of US cat food samples by brand (A) and price (B).

There was a high variance of samples from the same brand, which was partly explained by some of the less expensive foods have higher Carbon isotope values, probably reflecting higher levels of corn, a C4 plant. 

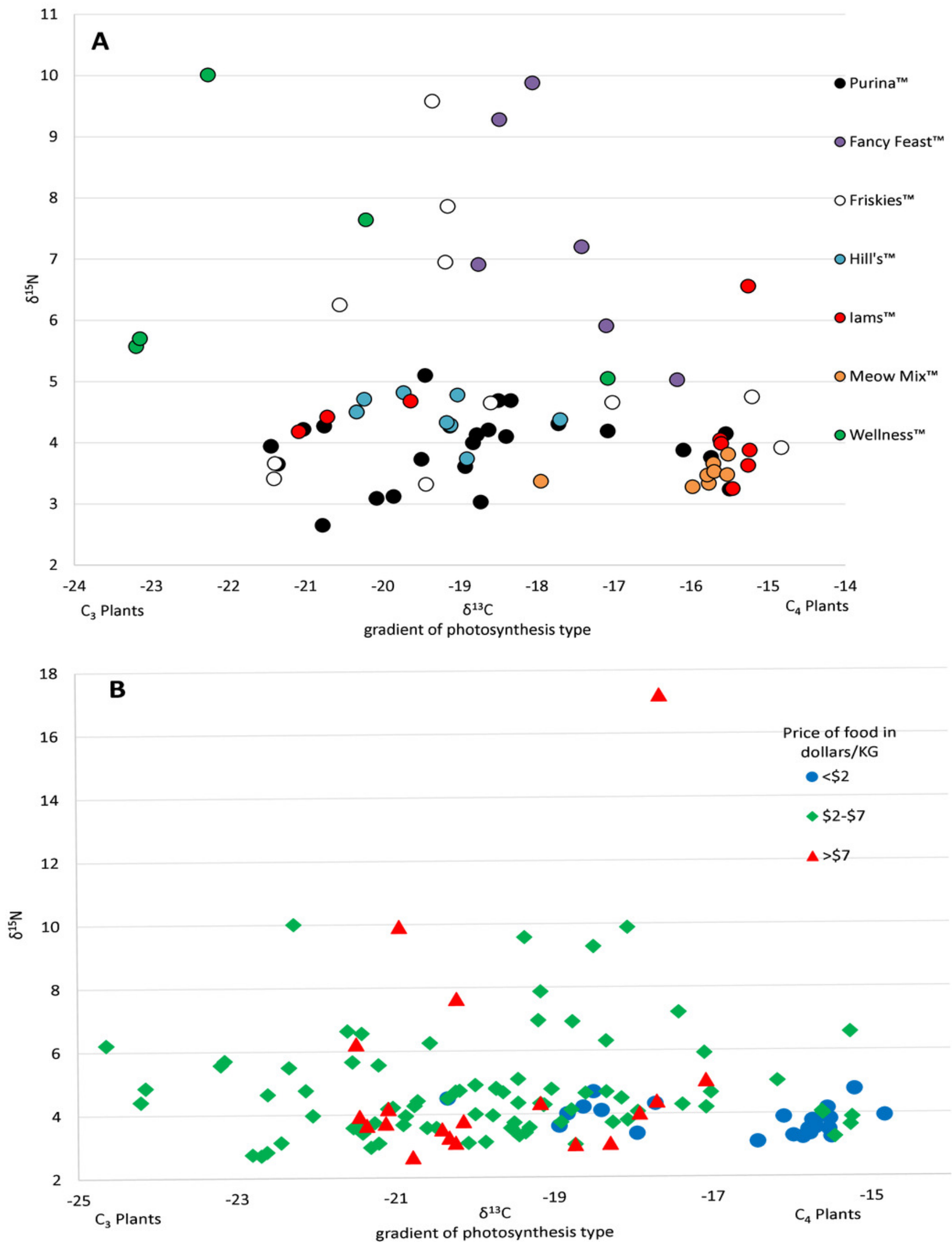


\section{Figure 3}

Classifications of cats by diet.

Cats were classified by comparing the isotope signatures from their hair to their pet food and potential native prey species. Of the 47 cats included in the analysis, 13 cats were classified as hunters (Fig. 3A), 7 cats were classified as non-hunters likely eating mostly pet food (Fig. 3B), 11 cats were classified as having relatively high carbon values compared to potential dietary inputs (Fig. 3C), and 16 cats were classified as having an uncertain diet (Fig. 3D). Characteristic individual isospace plots for each category of cat are shown.
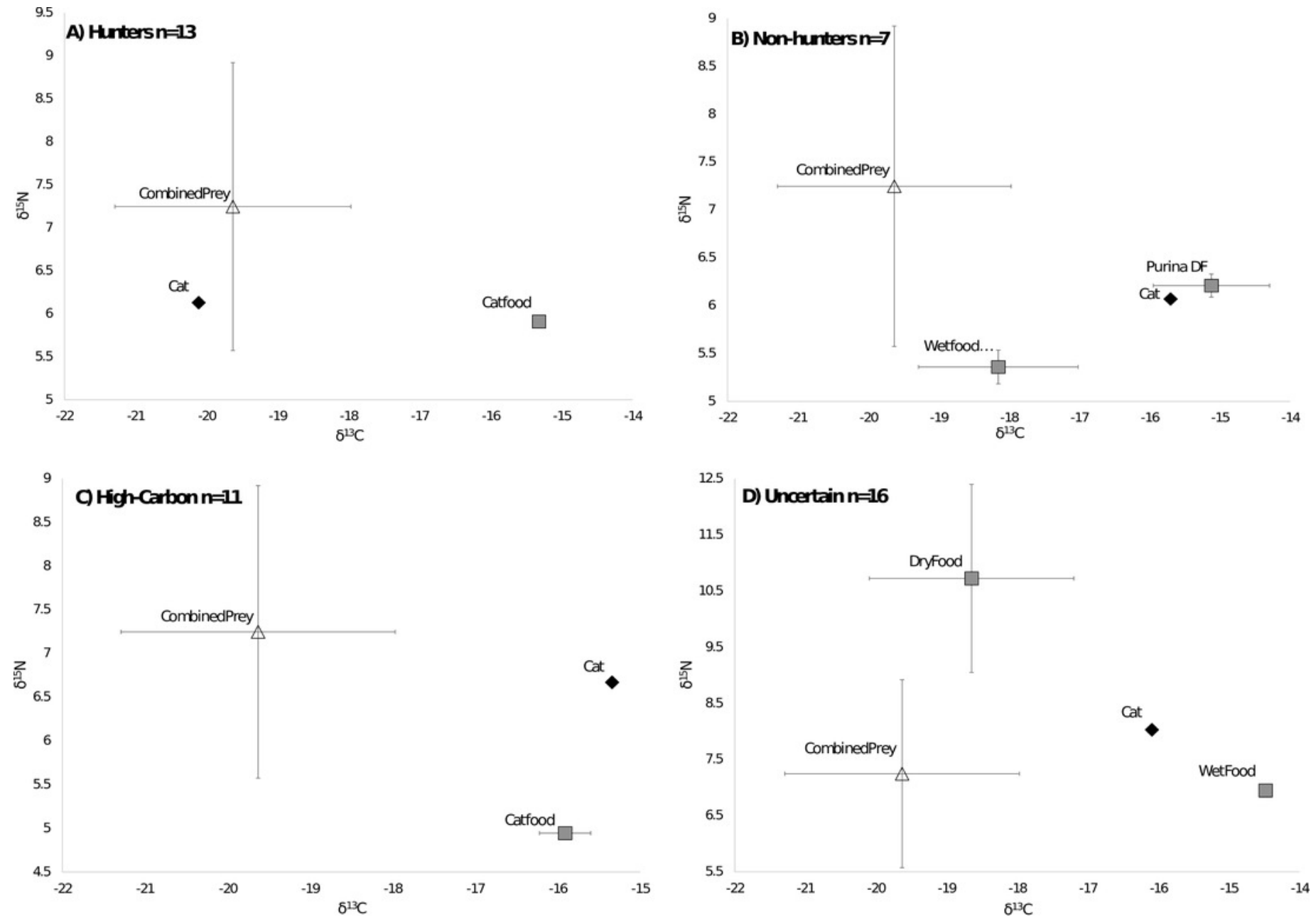
Figure 4

Isotope values for 47 cats classified by diet category.

All 47 cats represented are from southeastern U.S. as mixing models were derived from cats in this region. By including the pet food, the models were able to detect wild prey in the diet of some individuals, but this plot shows broad overlap of these categories in isospace, due to the high variability of the isotopic values in pet food.

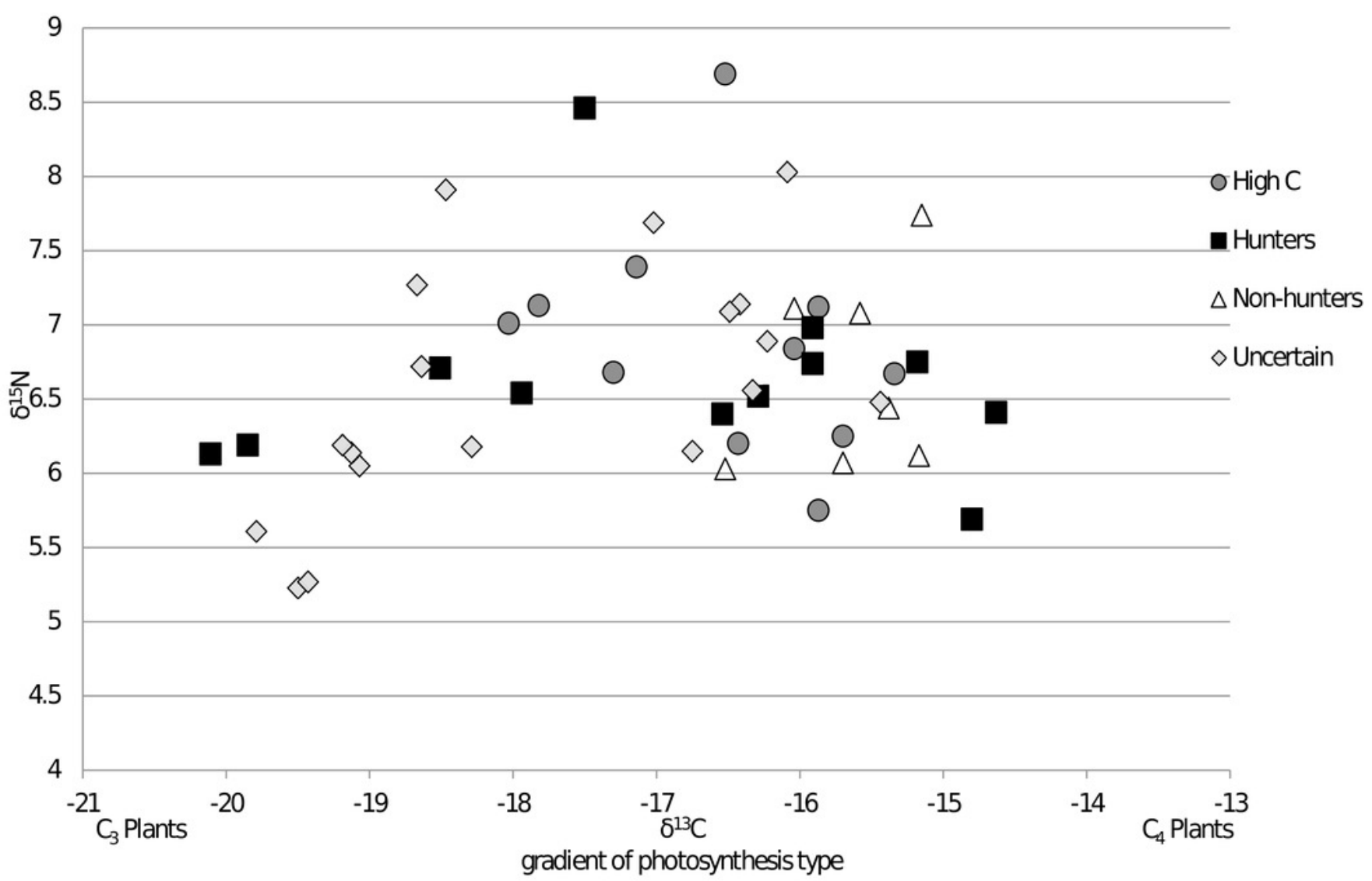

